\title{
An Optimized Modal Balancing Approach for a Flexible Rotor Using a Vibration Response While the Rotor Is Speeding Up
}

\author{
Liqing Li $\mathbb{D},,^{1,2,3}$ Yuanhang Hou $\mathbb{D},,^{1,2}$ and Shuqian Cao $\mathbb{D}^{1,2}$ \\ ${ }^{1}$ Department of Mechanics, Tianjin University, Tianjin 300354, China \\ ${ }^{2}$ Tianjin Key Laboratory of Nonlinear Dynamics and Control, Tianjin 300354, China \\ ${ }^{3}$ National Demonstration Center for Experimental Mechanics Education (Tianjin University), Tianjin 300354, China
}

Correspondence should be addressed to Shuqian Cao; sqcao@tju.edu.cn

Received 1 September 2021; Revised 3 January 2022; Accepted 5 February 2022; Published 7 March 2022

Academic Editor: Shan Gao

Copyright (C) 2022 Liqing Li et al. This is an open access article distributed under the Creative Commons Attribution License, which permits unrestricted use, distribution, and reproduction in any medium, provided the original work is properly cited.

\begin{abstract}
The modal balancing method (MBM) is an effective method for reducing the vibration caused by the unbalancing of a rotor. A rotor's modal parameters, especially its modal shapes, need to be accurately calculated in this method. This paper proposes an optimized modal balancing approach for flexible rotors. The vibration modes of the rotor are first obtained with experimental modal analysis based on the rotor's response signals while the rotor is speeding up. The rotor balancing strategy is subsequently optimized by the sensitivity analysis of the mode shapes. Orthogonal trial masses are obtained based on the orthogonality of each vibration mode, and the correction masses are finally calculated by using the influence coefficients of the trial masses. An experimental result is shown to demonstrate and validate that the proposed approach is able to achieve superior accuracy compared to the conventional MBM.
\end{abstract}

\section{Introduction}

A rotor is the core component of rotating machinery. The main vibration of rotating equipment is caused by the unbalance of a rotor. Rigid rotors were used in early rotating machinery. The working speed of these rotors was far lower than their critical speed, and hence, the rotors did not produce large bending deformations [1]. With the growing demand for power, flexible rotors have been increasingly used in rotating machineries, such as in gas turbines and aerospace engines. The working speed of these rotors far exceeds the first critical speed and is even higher than the second and third critical speeds [2]. When passing through different critical speeds, a rotor will show different forms of bending deformation. The deformation at the critical speed will change the initial unbalanced distribution of a rotor and even cause additional unbalance. Therefore, flexible rotors must be accurately balanced to safely pass through each critical speed to the designed speed $[3,4]$.

Generally, rotor balancing methods include the influence coefficient method (ICM), modal balancing method (MBM), and comprehensive balancing method [5-10]. The ICM obtains the influence coefficient by adding trial masses to different correction planes of the rotor in trial runs [11-13]. Because each influence coefficient is measured via experiments, a significant number of revolutions are required to obtain the correction data at the balancing speed. Turbomachinery users are constantly concerned about the increased cost and possible downtime associated with the ICM due to its limitations and difficulties in flexible rotor balancing $[14,15]$. In addition, the selection of balancing planes is heavily dependent on the operator's experience. The lack of rotor dynamic characteristics may lead to balance failure [16-18]. Therefore, the application of the ICM in flexible rotor balancing is seriously restricted.

The MBM is a method based on flexural deformation for an unbalanced rotor. The unbalance can be expressed in the form of each vibration mode, and the rotor deflection of each mode can be excited by the corresponding vibration unbalance. The method generally assumes that the rotor system has a plane vibration mode. Balancing one mode will not affect any other modes and the effects of higher 
modes are not considered. Grobel [19] proposed a balancing technique similar to MBM for turbine rotors. Kellenberger [20] performed an in-depth study of the MBM to continuously improve the theory of balancing flexible rotors with the MBM and proposed an $N$-plane method and an $N+2$ planes method for flexible rotor balancing. Determining the mode shapes of a rotor is a key step for modal balancing, and these shapes are generally calculated with the finite element (FE) method. Bertoneri [21] developed a dynamical balancing system by using the FE model to calculate the rotor vibration mode for the correction masses in balancing. The FE model was relatively simple in the balancing system. It was only applicable to simply supported rotors. Prior to balancing a rotor, the structural parameters of the rotor need to be imported into the FE model. Saldarriaga [22] regarded the balancing of a flexible rotor as a process for solving two types of inverse problems. The first inverse problem is used to obtain dynamical characteristics (modal parameters) of a rotor system and the second inverse problem is used to determine the unbalance. The FE method is also used to obtain the rotor modal parameters, while the parameters of the pedestals are identified using the frequency response function (FRF). Khulief [23] adopted a balancing method and developed a hybrid experiment and analysis technique to balance a highspeed flexible rotor. The technology combines FE modeling, experimental modal analysis, vibration measurement, and mathematical identification. Li [24] proposed a trialless rebalancing method based on the FE model. This method uses the vibration response and the accurate FE model of a rotor system to obtain the correction masses of a rotor. These methods without trial masses eliminate trial runs in rotor balancing to save time [25]. In most MBMs, a highly accurate FE model of a rotor is required to obtain the modal information. Sinha [26] found that the maximum error in the estimated unbalance amplitude could reach up to $45 \%$ for simultaneous random errors of $5 \%$ in the FE model of a rotor. It is always difficult to obtain accurate FE models for complex rotors. This difficulty will be obvious for the operator when balancing various rotors. Additionally, it is very dangerous in most MBMs to run a rotor stably at a critical speed.

In this paper, an optimized MBM is proposed for balancing flexible rotors. The procedure of the approach is shown in Figure 1. Firstly, the modal parameters of a rotor, such as the mode shapes and critical speeds, are calculated by using the vibration response of the rotor while the rotor is speeding up. Secondly, the modal parameters are used to calculate the orthogonal trial masses and calculate the sensitivity. Thirdly, the mode shape sensitivity analysis for the local mass changes of the rotor is used to optimize the balancing strategy in selecting correction planes, and the correction masses are calculated by using the MBM. Finally, experimental validation is conducted to assess the achievable accuracy of the proposed method and the conventional MBM.

\section{Modal Parameter Estimation for the Rotor}

An isotropic rotor is considered to be a multidegree of freedom linear vibration system. The generalized

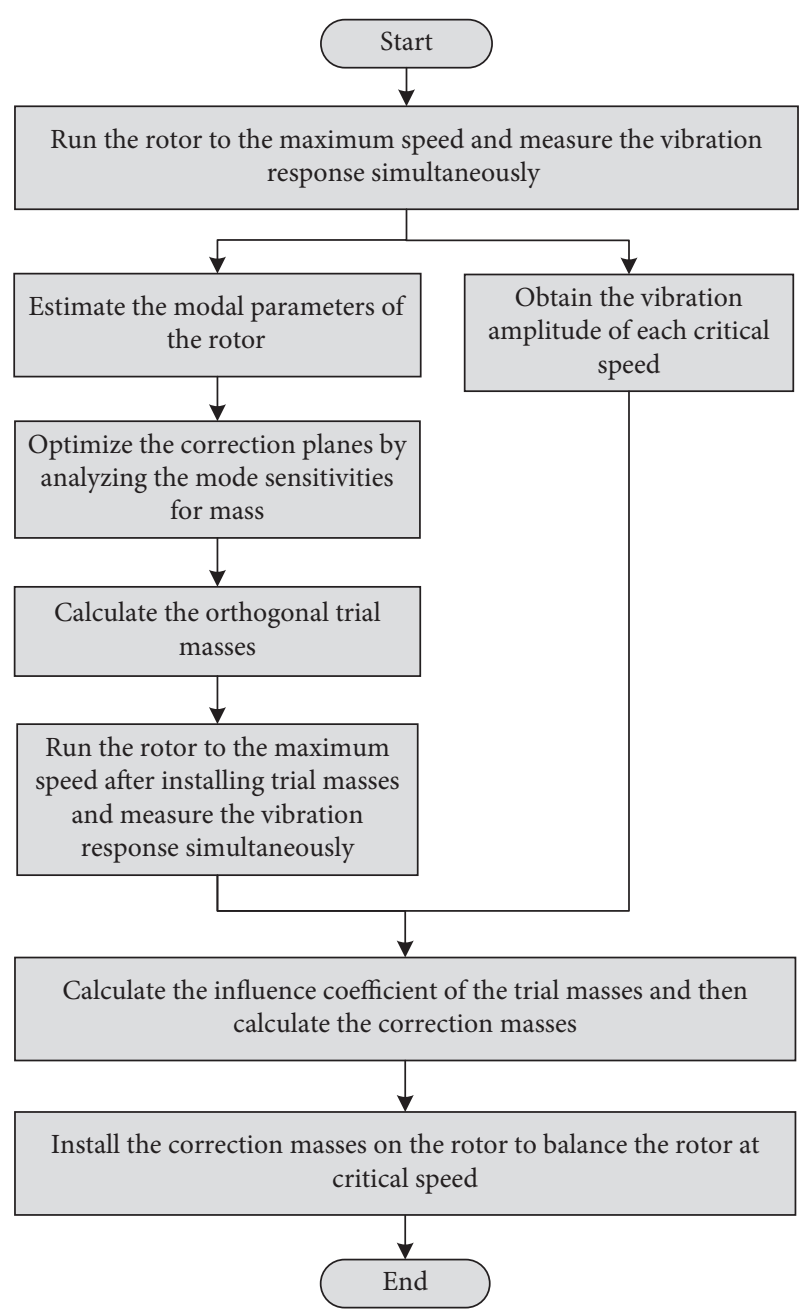

FIGURE 1: The schematic illustration of the approach.

complex coordinate expression of its motion equation is shown in equation (1) [27]. The gyroscopic effect of the rotor disc has a great influence on the dynamics of the rotor system, especially for large rotors. For most rotors, due to the existence of the gyroscopic effect, the Campbell diagram of the system is not as "flat" as the Jeffcott rotor.

$$
\mathbf{M} \ddot{q}(t)+(\Omega \mathbf{G}+\mathbf{C}) \dot{q}(t)+\mathbf{K q}(t)=\mathbf{f}(t),
$$

where $\mathbf{M}, \mathbf{C}$, and $\mathbf{K}$ are the mass, stiffness, and damping matrices of the rotor system, respectively, and they are independent of the rotational speed of the rotor; $\mathbf{G}$ is the gyroscopic matrix of the rotor, which is dependent on the rotational speed of the rotor; $\mathbf{q}(t)$ is the dimensional generalized coordinate vector; $\mathbf{f}(t)$ is the corresponding generalized force vector. The FRF matrix of the rotor system described by (1) results from the inversion of the impedance matrix. It can be expressed as follows:

$$
\mathbf{H}(\omega)=\left.\mathbf{Z}(s)^{-1}\right|_{s=j \omega}=\left(\mathbf{K}+j(\Omega \mathbf{G}+\mathbf{C}) \omega-\mathbf{M} \omega^{2}\right)^{-1},
$$

where $\mathbf{Z}(s)$ is the impedance matrix of the rotor system, which can be obtained by Laplace transform. The FRF for the 
two physical coordinates $k$ and $s$ can be expressed using the residue as follows:

$$
\begin{aligned}
H_{k s}(\omega) & =\sum_{r=1}^{N}\left(\frac{R_{k s}^{(r)}}{j \omega-\lambda_{r}}+\frac{R_{k s}^{(r) *}}{j \omega-\lambda_{r}^{*}}\right) \\
& =\sum_{r=1}^{N}\left(\frac{a_{r} \varphi_{k}^{(r)} \varphi_{s}^{(r)}}{j \omega-\lambda_{r}}+\frac{a_{r}^{*} \varphi_{k}^{(r) *} \varphi_{s}^{(r) *}}{j \omega-\lambda_{r}^{*}}\right) \\
& =\sum_{r=1}^{2 N} \frac{a_{r} \varphi_{k}^{(r)} \varphi_{s}^{(r)}}{j \omega-\lambda_{r}}
\end{aligned}
$$

where $*$ represents the conjugation and $\lambda_{r}$ is the pole of the system. $\lambda_{r}=-\sigma_{r}+j \omega_{r}$ and $\lambda_{r}{ }^{*}=-\sigma_{r}-j \omega_{r}$, where $\sigma_{r}$ is the attenuation coefficient, $\omega_{r}$ is the modal frequency, $N$ is the number of modes, $a_{r}$ is the modal scale factor for mode $r$, and $\varphi_{k}^{(r)}$ and $\varphi_{s}^{(r)}$ represent the components of $r$-th mode shape vectors of the rotor at the coordinates $k$ and $s$, respectively.

Generally, the initial unbalanced mass distributions of a rotor are generated during rotor manufacturing. The initial distributions are always unknown. Since the unbalance distributions can be expressed in the form of vibration modes, $n$ modal unbalances can be obtained, and these can be written as the column vector $\mathbf{u}=\left[\begin{array}{llll}u_{1} & u_{2} & \cdots & u_{n}\end{array}\right]^{T}$. The excitation force generated by the unbalancing of the rotor is as follows:

$$
\mathbf{f}(t)=\mathbf{u} \Omega^{2} e^{j \Omega t} .
$$

For the case of $\omega=\Omega$, the Fourier transform of the unbalanced force of the rotor system can be expanded as follows:

$$
\mathbf{f}(\omega)=\frac{1}{T} \int_{-T / 2}^{T / 2} \mathbf{u} \Omega^{2} e^{j \Omega t} e^{-j \omega t} \mathrm{~d} t=\mathbf{u} \omega^{2} .
$$

The vibration of the $k$-th point on the rotor is a superposition response by $S$ excitation forces. It can be written as follows:

$$
\begin{aligned}
x_{k}(\omega) & =\sum_{s=1}^{S} H_{k s}(\omega) f_{s}(\omega) \\
& =\sum_{s=1}^{S} H_{k s}(\omega) u_{s} \omega^{2} .
\end{aligned}
$$

The ratio of the response at testing point $k$ to the response at testing point $p$ on a structure is defined as the transfer rate (TR) from testing point $p$ to testing point $k$, where the testing point $p$ is the reference point. If the modes of the rotor system can be effectively separated, it means that the coupling between each mode is small, and the response of the system will be dominated by the $r$-th modal vibration at the modal frequency $\omega_{r}$. The contribution of the other modes at this frequency can be ignored. The TR at the critical speed $\omega_{r}$ can be written as follows:

$$
\begin{aligned}
& \frac{x_{k}(\omega)}{x_{p}(\omega)} \approx \frac{u_{r} \omega^{2} \sum_{s=1}^{S} H_{k s}(\omega)}{u_{r} \omega^{2} \sum_{s=1}^{S} H_{p s}(\omega)} \\
&=\frac{\sum_{s=1}^{S} \sum_{r=1}^{2 N}\left(a_{r} \varphi_{k}^{(r)} \varphi_{s}^{(r)} / j \omega-\lambda_{r}\right)}{\sum_{s=1}^{S} \sum_{r=1}^{2 N}\left(a_{r} \varphi_{p}^{(r)} \varphi_{s}^{(r)} / j \omega-\lambda_{r}\right)} \\
& \approx \frac{\sum_{s=1}^{S}\left(a_{r} \varphi_{k}^{(r)} \varphi_{s}^{(r)} / j \omega-\lambda_{r}\right)}{\sum_{s=1}^{S}\left(a_{r} \varphi_{k}^{(r)} \varphi_{s}^{(r)} / j \omega-\lambda_{r}\right)} \\
&=\frac{\varphi_{k}^{(r)}}{\varphi_{p}^{(r)}} .
\end{aligned}
$$

The rotational speed can be measured accurately. The vibration response at each test point on the rotor can be measured in the integer period $(T)$ of the rotational speed. For the finite length signal $x(t) \mid t \in[0, T]$, the finite Fourier transform is $x(\omega)=\int_{0}^{T} x(t) e^{-j \omega t} \mathrm{~d} t$. With this definition, the autopower spectrum and the cross-power spectrum of the two signals $x_{k}(t)$ and $x_{p}(t)$ are defined as follows:

$$
\left\{\begin{array}{l}
G_{p p}\left(\omega_{r}\right)=\frac{1}{T} x_{p}^{*}\left(\omega_{r}\right) x_{p}\left(\omega_{r}\right), \\
G_{p k}\left(\omega_{r}\right)=\frac{1}{T} x_{p}^{*}\left(\omega_{r}\right) x_{k}\left(\omega_{r}\right) .
\end{array}\right.
$$

Combining (7) and (8), we arrive at the following:

$$
\frac{\varphi_{k}^{(r)}}{\varphi_{p}^{(r)}}=\frac{x_{k}\left(\omega_{r}\right)}{x_{p}\left(\omega_{r}\right)}=\frac{G_{p k}\left(\omega_{r}\right)}{G_{p p}\left(\omega_{r}\right)} .
$$

It is assumed that the rotor system is linear. In the power spectrum amplitude curve, the frequency corresponding to every peak value is the critical speed of a rotor. The power spectrum of each testing position on the rotor should contain the same peak frequency unless the testing position is the modal node of a rotor. According to (9), the ratio of the $r$-th mode shape component of testing point $k$ to testing point $p$ is equal to the ratio of the cross-power spectrum $G_{p k}(\omega)$ to the autopower spectrum $G_{p p}(\omega)$ at the critical speed. $\varphi_{k}^{(r)} / \varphi_{p}^{(r)}$ is complex, and it contains the magnitude and the phase angle. This can be regarded as the normalized mode shape with reference point $p$.

Since the excitation forces are unmeasurable, only the response spectrum can be used to determine the modal parameters of the rotor. It is necessary to synthesize the response spectrum of all the measuring points and the structural characteristics to comprehensively determine the modal parameters. 


\section{Correction Plane Optimization with Sensitivity Analysis}

Assuming

$$
\begin{aligned}
\mathbf{P} & =\left[\begin{array}{cc}
\Omega \mathbf{G} & \mathbf{M} \\
\mathbf{M} & 0
\end{array}\right], \\
\mathbf{Q} & =\left[\begin{array}{cc}
\mathbf{K} & 0 \\
0 & -\mathbf{M}
\end{array}\right], \\
q^{\prime}(t) & =\left[\begin{array}{c}
\mathbf{q}(t) \\
\dot{q}(t)
\end{array}\right] .
\end{aligned}
$$

The state space equation of the rotor system described by (1) can be written as follows [28]:

$$
\mathbf{P} \dot{q}^{\prime}(t)+\mathbf{Q} q^{\prime}(t)=0 .
$$

The eigenvalues and eigenvectors in the state space are set to $\lambda_{i}, \Psi^{(i)}$, and $\mathbf{P}$ and $\mathbf{Q}$ are assumed to be the coefficient matrices of the state equation. From the orthogonality of the eigenvectors, the following expression is obtained:

$$
\begin{aligned}
& \Psi^{(k) T} \mathbf{P} \Psi^{(i)}= \begin{cases}0, & (i \neq k), \\
1, & (i=k),\end{cases} \\
& \Psi^{(k) T} \mathbf{Q} \Psi^{(i)}= \begin{cases}0, & (i \neq k), \\
-\lambda_{i}, & (i=k),\end{cases}
\end{aligned}
$$

in which $\Psi^{(i)}$ has been normalized according to certain conditions.

From the generalized eigenvalue problem of the state space $(\lambda \mathbf{P}+\mathbf{Q}) \Psi=0$, the following expression is obtained:

$$
\lambda_{i} \mathbf{P} \Psi^{(i)}+\mathbf{Q} \Psi^{(i)}=0
$$

Calculating the partial derivative of the local mass $m$ gives the following:

$$
\frac{\partial \lambda_{i}}{\partial m} \mathbf{P} \Psi^{(i)}+\lambda_{i} \frac{\partial \mathbf{P}}{\partial m} \Psi^{(i)}+\lambda_{i} \mathbf{P} \frac{\partial \Psi^{(i)}}{\partial m}+\frac{\partial \mathbf{Q}}{\partial m} \Psi^{(i)}+\mathbf{Q} \frac{\partial \Psi^{(i)}}{\partial m}=0 .
$$

Multiplying $\Psi^{(k) T}$ by the left side of (15) gives the following:

$$
\frac{\partial \lambda_{i}}{\partial m} \Psi^{(k) T} P \Psi^{(i)}+\Psi^{(k) T}\left(\lambda_{i} \frac{\partial \mathbf{P}}{\partial m}+\frac{\partial \mathbf{Q}}{\partial m}\right) \Psi^{(i)}+\left(\lambda_{i} \Psi^{(k) T} \mathbf{P}+\Psi^{(k) T} \mathbf{Q}\right) \frac{\partial \Psi^{(i)}}{\partial m}=0
$$

From (14), the following expression is obtained:

$$
\lambda_{i} \Psi^{(i) T} \mathbf{P}+\Psi^{(i) T} \mathbf{Q}=0 .
$$

When $i \neq k$, from equations (12), (16), and (17), the following expression is obtained:

$$
\left(\lambda_{i}-\lambda_{k}\right) \Psi^{(k) T} \mathbf{P} \frac{\partial \Psi^{(i)}}{\partial m}+\Psi^{(k) T}\left(\lambda_{i} \frac{\partial \mathbf{P}}{\partial m}+\frac{\partial \mathbf{Q}}{\partial m}\right) \Psi^{(i)}=0 .
$$

For eigenvectors $\Psi^{(s)}$ constituting a set of bases of $N$ dimensional vector space, $\partial \Psi^{(i)} / \partial m$ is a linear combination of the eigenvectors:

$$
\frac{\partial \Psi^{(i)}}{\partial m}=\sum_{s=1}^{n} a_{s} \Psi^{(s)}
$$

Substituting (19) into (18) and considering (12) yields

$$
a_{k}=\frac{1}{\lambda_{k}-\lambda_{i}} \boldsymbol{\Psi}^{(k) T}\left(\lambda_{i} \frac{\partial \mathbf{P}}{\partial m}+\frac{\partial \mathbf{Q}}{\partial m}\right) \Psi^{(i)}, \quad(k \neq i) .
$$

Substituting (10) into (20), the following expression is obtained:

$$
\begin{aligned}
a_{k} & =\frac{1}{\lambda_{k}-\lambda_{i}} \Phi^{(k) T}\left(\lambda_{i}^{2} \frac{\partial \mathbf{M}}{\partial m}+\lambda_{i} \frac{\partial \Omega \mathbf{G}}{\partial m}+\frac{\partial \mathbf{K}}{\partial m}\right) \Phi^{(i)} \\
& =\frac{\lambda_{i}^{2}}{\lambda_{k}-\lambda_{i}} \Phi^{(k) T} \boldsymbol{\Phi}^{(i)}, \quad(k \neq i) .
\end{aligned}
$$

When $i=k$, the partial derivative of (12) is calculated directly, and considering the symmetry of $\mathbf{P}$, the following expression is obtained:

$$
2 \Psi^{(k) T} \mathbf{P} \frac{\partial \Psi^{(i)}}{\partial m}+\Psi^{(k) T} \frac{\partial \mathbf{P}}{\partial m} \Psi^{(i)}=0 .
$$

Substituting (19) into (22) and considering (12), the following expression is obtained:

$$
a_{k}=a_{i}=-\frac{1}{2} \Psi^{(i) T} \frac{\partial \mathbf{P}}{\partial m} \Psi^{(i)}, \quad(i=k) .
$$

In the same way as (21), by substituting (10) into (2) 3, the following expression is obtained:

$$
\begin{aligned}
a_{k} & =a_{i}=-\frac{1}{2} \Phi^{(i) T}\left(2 \lambda_{i} \frac{\partial \mathbf{M}}{\partial m}+\frac{\partial \Omega \mathbf{G}}{\partial m}\right) \Phi^{(i)} \\
& =-\lambda \Phi^{(i) 2}, \quad(k=i) .
\end{aligned}
$$

The mode shape sensitivity of the rotor for mass changes is defined as follows:

$$
\begin{aligned}
S(\boldsymbol{\Phi}, m) & =\frac{\partial \boldsymbol{\Phi}^{(i)}}{\partial m} \\
& =\sum_{k=1}^{2 n} a_{k} \boldsymbol{\Phi}^{(k)} .
\end{aligned}
$$

In which $a_{k}$ can be obtained from (21) and (24). It can be written in more detail as follows: 


$$
\begin{aligned}
\text { when } k=i a_{k} & = \begin{cases}-2 \lambda_{i} \varphi_{e}^{(i)} \varphi_{f}^{(i)}, & (e \neq f), \\
-\lambda_{i} \varphi_{e}^{(i) 2}, & (e=f) .\end{cases} \\
\text { when } k \neq i: a_{k} & = \begin{cases}\frac{\lambda_{i}^{2}}{\lambda_{k}-\lambda_{i}}\left(\varphi_{e}^{(k)} \varphi_{f}^{(i)}+\varphi_{e}^{(i)} \varphi_{f}^{(k)}\right), & (e \neq f), \\
\frac{\lambda_{i}^{2}}{\lambda_{k}-\lambda_{i}} \varphi_{e}^{(k)} \varphi_{e}^{(i)}, & (e=f) .\end{cases}
\end{aligned}
$$

In (26) and (27), $\lambda_{i}=2 \pi f_{i}$ is the $i$-th eigenvalue of the rotor. $\varphi$ is the component of the eigenvector. $i$ and $k$ represent the modal numbers. $e$ and $f$ represent the testing points.

From the foregoing analysis, the mode shape sensitivity of the rotor for the mass changes can be expressed as a linear combination of the mode shapes, where $a_{k}$ is the weight of the $k$-th mode. By analyzing the mode shape sensitivities for the local mass, the balancing strategy can be optimized by selecting the appropriate correction planes.

\section{The Optimized Modal Balancing Approach}

According to the $\mathrm{N}$-plane rotor balancing theory, $\mathrm{N}$ correction planes are required to balance $N$ modal unbalances [20]. The balancing condition is as follows:

$$
\left[\begin{array}{cccc}
\varphi_{x_{1}}^{(1)} & \varphi_{x_{2}}^{(1)} & \cdots & \varphi_{x_{N}}^{(1)} \\
\varphi_{x_{1}}^{(2)} & \varphi_{x_{2}}^{(2)} & \cdots & \varphi_{x_{N}}^{(2)} \\
\vdots & \vdots & \vdots & \\
\varphi_{x_{1}}^{(N)} & \varphi_{x_{2}}^{(N)} & \cdots & \varphi_{x_{N}}^{(N)}
\end{array}\right]\left[\begin{array}{cccc}
U_{1}\left(x_{1}\right) & U_{2}\left(x_{1}\right) & \cdots & U_{N}\left(x_{1}\right) \\
U_{1}\left(x_{2}\right) & U_{2}\left(x_{2}\right) & \cdots & U_{N}\left(x_{2}\right) \\
\vdots & \vdots & \vdots & \\
U_{1}\left(x_{N}\right) & U_{2}\left(x_{N}\right) & \cdots & U_{N}\left(x_{N}\right)
\end{array}\right]=-\left[\begin{array}{cccc}
u_{1} & 0 & \cdots & 0 \\
0 & u_{2} & \cdots & 0 \\
\vdots & \vdots & \vdots & \\
0 & 0 & \cdots & u_{N}
\end{array}\right]
$$

where $\varphi_{x_{N}}^{(N)}$ is the component of the mode shapes of the rotor, the subscript $x_{N}$ is the correction plane, and $N$ is the number of modes, as well as the number of critical speeds, to be balanced. $U_{N}\left(x_{N}\right)$ is the component of the corrections matrix. $u_{N}$ and $U_{N}\left(x_{N}\right)$ are complex and include the correction mass and the correction phase mass. $u_{N}$ is the $N$-th component of the modal unbalance, which is equivalent to the initial mass unbalance distribution of the rotor.

To ensure that the correction of each mode only affects the current mode shape, the correction value vector must be orthogonal. Each column of the correction matrix is a set of mass corrections installed on correction planes. As can be seen from (28), each column of the correction matrix is a set of mass corrections installed on the correction planes that only balance the corresponding modal unbalance and do not affect the others.

It is assumed that the trail masses $\mathbf{W}_{i}=\left[\begin{array}{llll}w_{i}\left(x_{1}\right) & w_{i}\left(x_{2}\right) & \cdots & w_{i}\left(x_{N}\right)\end{array}\right]^{T}$ of each mode on the correction plane satisfy modal orthogonality:

$$
\left[\begin{array}{cccc}
\varphi_{x_{1}}^{(1)} & \varphi_{x_{2}}^{(1)} & \cdots & \varphi_{x_{N}}^{(1)} \\
\varphi_{x_{1}}^{(2)} & \varphi_{x_{2}}^{(2)} & \cdots & \varphi_{x_{N}}^{(2)} \\
\vdots & \vdots & \vdots & \\
\varphi_{x_{1}}^{(N)} & \varphi_{x_{2}}^{(N)} & \cdots & \varphi_{x_{N}}^{(N)}
\end{array}\right]\left[\begin{array}{cccc}
w_{1}\left(x_{1}\right) & w_{2}\left(x_{1}\right) & \cdots & w_{N}\left(x_{1}\right) \\
w_{1}\left(x_{2}\right) & w_{2}\left(x_{2}\right) & \cdots & w_{N}\left(x_{2}\right) \\
\vdots & \vdots & \vdots & \\
w_{1}\left(x_{N}\right) & w_{2}\left(x_{N}\right) & \cdots & w_{N}\left(x_{N}\right)
\end{array}\right]=\mathbf{I}
$$

where all the mode shapes have been obtained with the method mentioned in Section 2. The trial masses matrix can be obtained via matrix inversion:

$$
\left[\begin{array}{cccc}
w_{1}\left(x_{1}\right) & w_{2}\left(x_{1}\right) & \cdots & w_{N}\left(x_{1}\right) \\
w_{1}\left(x_{2}\right) & w_{2}\left(x_{2}\right) & \cdots & w_{N}\left(x_{2}\right) \\
\vdots & \vdots & \vdots & \\
w_{1}\left(x_{N}\right) & w_{2}\left(x_{N}\right) & \cdots & w_{N}\left(x_{N}\right)
\end{array}\right]=\left[\begin{array}{cccc}
\varphi_{x_{1}}^{(1)} & \varphi_{x_{2}}^{(1)} & \cdots & \varphi_{x_{N}}^{(1)} \\
\varphi_{x_{1}}^{(2)} & \varphi_{x_{2}}^{(2)} & \cdots & \varphi_{x_{N}}^{(2)} \\
\vdots & \vdots & \vdots & \\
\varphi_{x_{1}}^{(N)} & \varphi_{x_{2}}^{(N)} & \cdots & \varphi_{x_{N}}^{(N)}
\end{array}\right]^{-1}
$$


Comparing (28) and (29), the following expression is obtained:

$$
\begin{aligned}
{\left[\begin{array}{c}
U_{i}\left(x_{1}\right) \\
U_{i}\left(x_{2}\right) \\
\vdots \\
U\left(x_{N}\right)
\end{array}\right] } & =-k_{i}\left[\begin{array}{c}
w_{i}\left(x_{1}\right) \\
w_{i}\left(x_{2}\right) \\
\vdots \\
w_{i}\left(x_{N}\right)
\end{array}\right] \\
\mathbf{U}_{i} & =-k_{i} \mathbf{W}_{i} .
\end{aligned}
$$

The trial masses are proportional to the correction masses. The multiples $k_{i}$ are obtained with trial runs that are equal to the modal unbalance $u_{i}$. From (28), the vibration caused by the initial unbalances and the correction masses of the rotor cancel each other out. In other words, the original vibration must be equal to the vibration caused by the correction masses in the rotor balancing. $u_{i}$ is unknown, but the proportionality constant $k_{i}$ can be obtained with an experiment. The total process of this approach is as follows:

Step 1. Speeding up the rotor to a speed that exceeds all critical speeds to be balanced. In this approach, due to the huge amplitude at the critical speed, the rotor should pass each critical speed quickly. The vibration data of the rotor during this process must be tested. The initial vibration of the unbalanced rotor is $V_{0 i}\left(x_{n}\right)$, where $i$ is the modal order of the rotor and $n$ the test point number of the rotor. Because the rotor is rotating at the critical speed, its response can be considered purely caused by a modal unbalance with the same order.

Step 2. Stopping the rotor. The trail masses corresponding to each critical speed should be installed on the rotor.

Step 3. Repeating Step 1. The vibration of the rotor $V_{1 i}\left(x_{j}\right)$ can be measured. At this time, the vibration is caused by both the initial unbalance and the trail masses. Then the following relationship can be established:

$$
\frac{V_{0 i}}{V_{1 i}-V_{0 i}}=\frac{U_{i}}{w_{i}}=-k_{i} \text {. }
$$

Step 4. The correction masses of each plane can be calculated with equation (32).

Since the trial masses are calculated based on the modal shapes of the rotor, theoretically, the ratio $k_{i}$ should be equivalent at any correction planes or corresponding testing points. In practice, the data for any of the vibration sensors can be chosen to calculate the correction masses unless the sensor is only arranged at the modal node. Additionally, the least square method can be used to optimize the data in the calculation.

\section{Experimental Validation}

5.1. The Rotor Experimental System. The rotor balancing experiment is carried out on a DHRMT rotor bench. The schematic diagram of the rotor system is shown in Figure 2, and a picture of the bench is shown in Figure 3. The bench is composed of a V-shaped pedestal, a speed-regulating motor, two bearings, an elastic coupling, a rotor shaft, three rotor discs, three eddy current sensors, and a photoelectric sensor. The bench is $810 \mathrm{~mm}$ long, $335 \mathrm{~mm}$ wide, and $133 \mathrm{~mm}$ high. The bench can be affixed to the ground with six feet. The rotor shaft has a length of $560 \mathrm{~mm}$ and a diameter of $10 \mathrm{~mm}$. In order to make the rotor work flexibly, two different dimensions of the rotor discs are designed and manufactured. Disc 1 and disc 3 have a diameter of $78 \mathrm{~mm}$, a thickness of $25 \mathrm{~mm}$, a weight of $800 \mathrm{~g}$, and a correction radius of $50 \mathrm{~mm}$. The diameter of disc 2 is $78 \mathrm{~mm}$, the thickness is $12.5 \mathrm{~mm}$, the weight is $400 \mathrm{~g}$, and the correction radius is $50 \mathrm{~mm}$. With the rotor discs installed, the span of the two bearings is $500 \mathrm{~mm}$. The three rotor discs divide the shaft into four segments, and the length of each segment is $125 \mathrm{~mm}$. The bench uses a speed-regulating motor to drive the shaft through an elastic coupling to rotate at different speeds. The maximum output power of the motor is $148 \mathrm{~W}$. The motor driver rectifies the $220 \mathrm{~V}$ AC power supply and outputs a PWM signal to drive the motor. The motor speed range is 0-6000 RPM.

A photoelectric sensor is installed on the side of the coupling with a bracket. A piece of a reflective paper is attached to the coupling, and the photoelectric sensor generates a pulse in a rotating circle. This pulse signal serves as a reference for the rotor speed and vibration phase. Three eddy current sensors are installed near the discs with the bracket to test the vertical vibration response of the shaft at the three discs. The technical parameters of the three eddy current sensors after calibration are shown in Table 1. A DH5922 N dynamic signal collector is used to collect the vibration signal of these sensors. The maximum sampling frequency of DH5922 $\mathrm{N}$ is $128 \mathrm{kHz}$, and the $\mathrm{A} / \mathrm{D}$ module is 24 bits.

The sensor number corresponds to the disc number. That is, sensor \# 1 tests the vibration of disc 1 , sensor \#2 tests the vibration of disc 2 , and sensor \#3 tests the vibration of disc 3. The three discs are also the correction planes. Threaded holes are set in the discs to mount the correction masses during balancing. These are shown in Figure 4 and 5.

\subsection{Modal Parameters of the Rotor Experimental System.} The motor speed of the bench shown in Figure 5 can only reach $6000 \mathrm{rpm}$, and it is difficult to reach the third critical speed of the rotor. Therefore, vibration testing and balancing are mainly carried out at the first and the second critical speeds. The rotor is dynamically balanced at a low speed to reduce the original imbalance of the rotor beforehand so that the rotor can pass the first two critical speeds.

As is generally known, the vibration of a structure is very large at resonance, and this is dangerous to a user. When the stress in the vibration exceeds the elastic stress limit of the structural material, it will cause permanent damage to the structure. The same is true for rotors. In a traditional MBM, a rotor runs at a certain critical speed constantly. The vibration response of the rotor will be too large to cause damage to the rotor shaft, bearing, or other parts. In addition, this is dangerous for operators. In the process of 


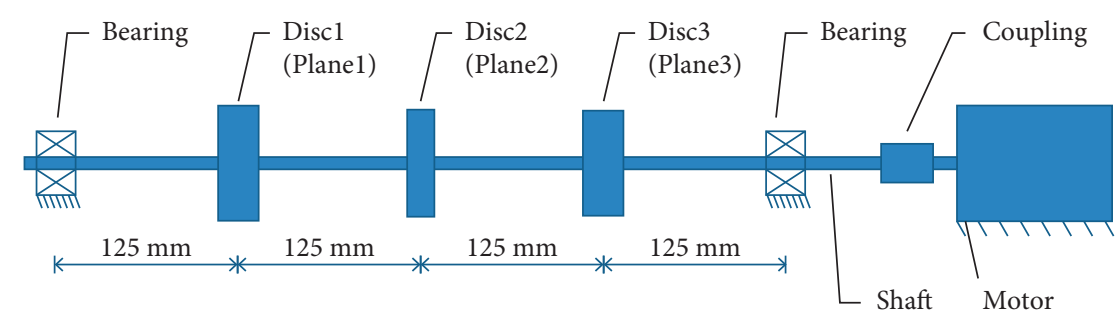

FIgURE 2: Schematic diagram of the rotor experimental system.

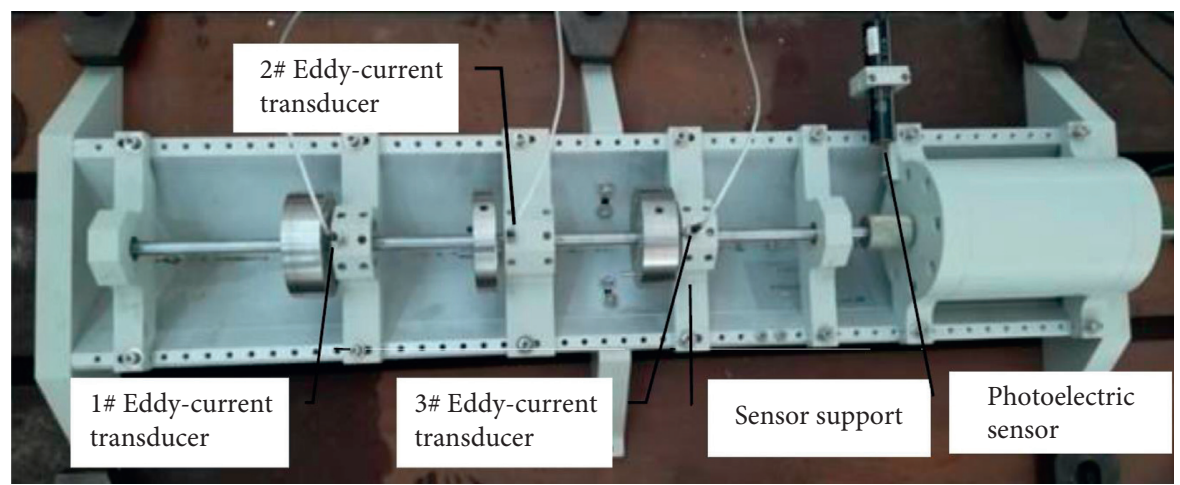

FIgURE 3: The setup of the rotor experimental system.

TABLE 1: Eddy current sensor parameters.

\begin{tabular}{lccc}
\hline Parameters & $\# 1$ & $\# 2$ & \\
\hline Measuring range (mm) & 1 & 1 & 1 \\
Sensitivity (V/mm) & 9.98 & 9.98 & 10.03 \\
Amplitude linearity & $0.21 \%$ & $0.60 \%$ & $0.40 \%$ \\
Amplitude repeatability & $0.33 \%$ & $0.64 \%$ & $0.37 \%$ \\
Zero error & $0.01 \%$ & $0.01 \%$ & $0.02 \%$ \\
Supply voltage (V) & \pm 15 & \pm 15 & \pm 15 \\
\hline
\end{tabular}

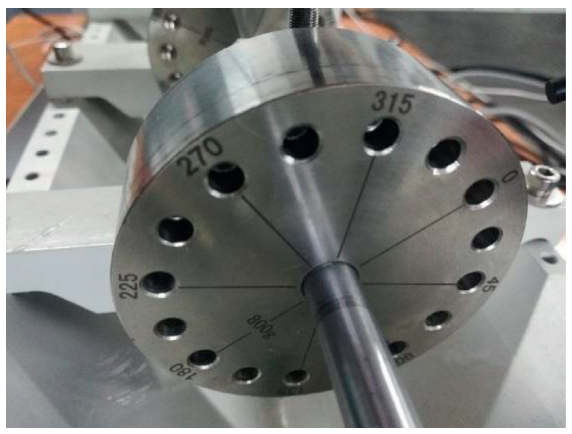

Figure 4: Disc with holes for mounting correction masses.

speeding up, the rotor can pass through the critical speed quickly without staying. The calculation of the modal parameters and correction masses using the vibration signals of a rotor while the rotor is speeding up is important not only for safety but also for efficiency and precision.

The vibration displacements of the three discs of the rotor before flexible balancing are shown in Figure 6. It can be seen from these curves that although the rotor can pass the two first critical speeds after low-speed balancing, the vibration displacements at the two critical speeds are still relatively large for the vibration mode excited by the corresponding unbalance. The maximum amplitude is $0.18 \mathrm{~mm}$.

Taking the vibration displacement on the first disc as the reference, the correlation functions of the vibration displacement for the three discs are as shown in Figures 7 and 8.

Three power spectrums are calculated as shown in Figures 7 and 8, and these spectrums can be used to identify the modal frequencies and the mode shapes. It can be 


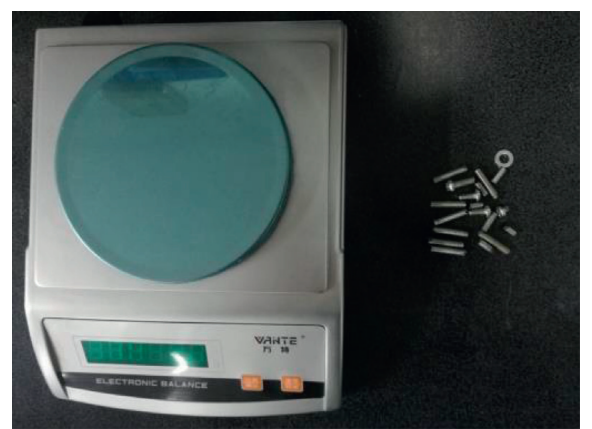

FIGURE 5: Electronic balance and small screws for correction masses.

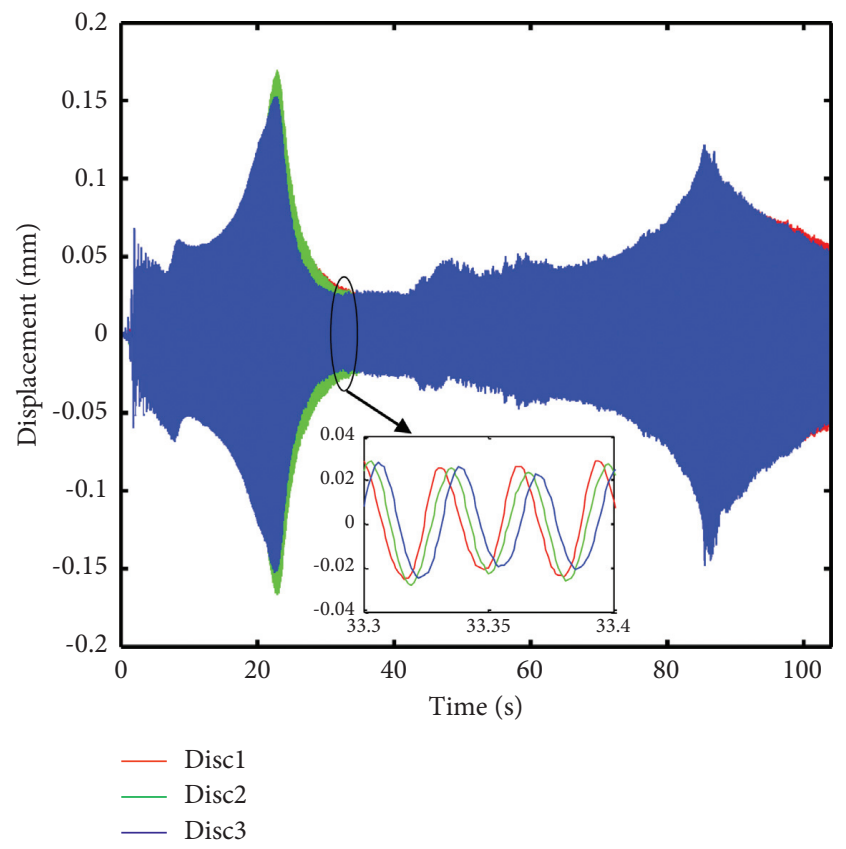

Figure 6: Vibration displacement of three discs while the rotor is speeding up.

determined that the first critical speed of the rotor is $23.44 \mathrm{~Hz}$, which corresponds to 1406.4 RPM. The second critical speed is $77.15 \mathrm{~Hz}$, which corresponds to $4629 \mathrm{RPM}$. From Figures 7 and 8, the mode shapes of the rotor listed in Table 2 can be obtained. These mode shapes have been normalized by the maximum. Figure 9 shows the mode shape of the rotor.

5.3. Sensitivity Analysis. The mode shape sensitivity data for the mass changes are calculated by using the method described in Section 3. The mode shapes of the rotor are shown in Figure 9. The calculation results are shown in Table 3. In the table, $m_{i}$ is the local mass in the $i$-th plane and $\varphi_{i}^{(r)}$ is the $r$-th mode shape component of the $i$-th plane.

Surveying the data horizontally for the first mode in Table 3, the absolute values of the sensitivity $\varphi_{2}^{(1)}$ for $m_{1}, m_{2}$ and $m_{3}$ in the first mode are larger. This implies that changing the three local masses has an obvious effect on the

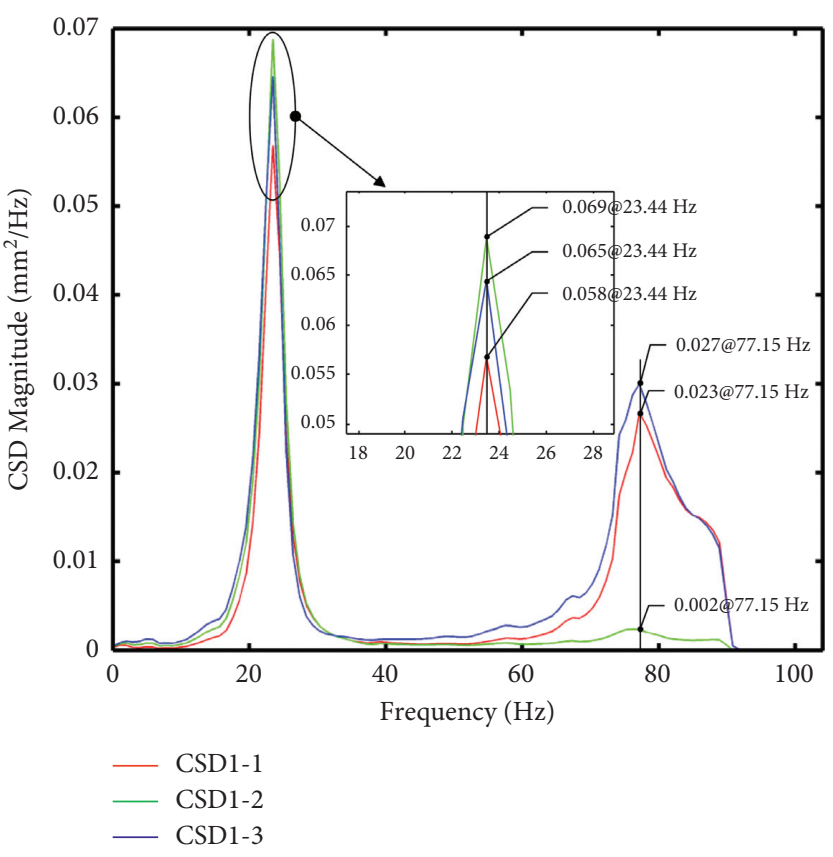

FIgURE 7: Magnitude curve of the power spectrum.

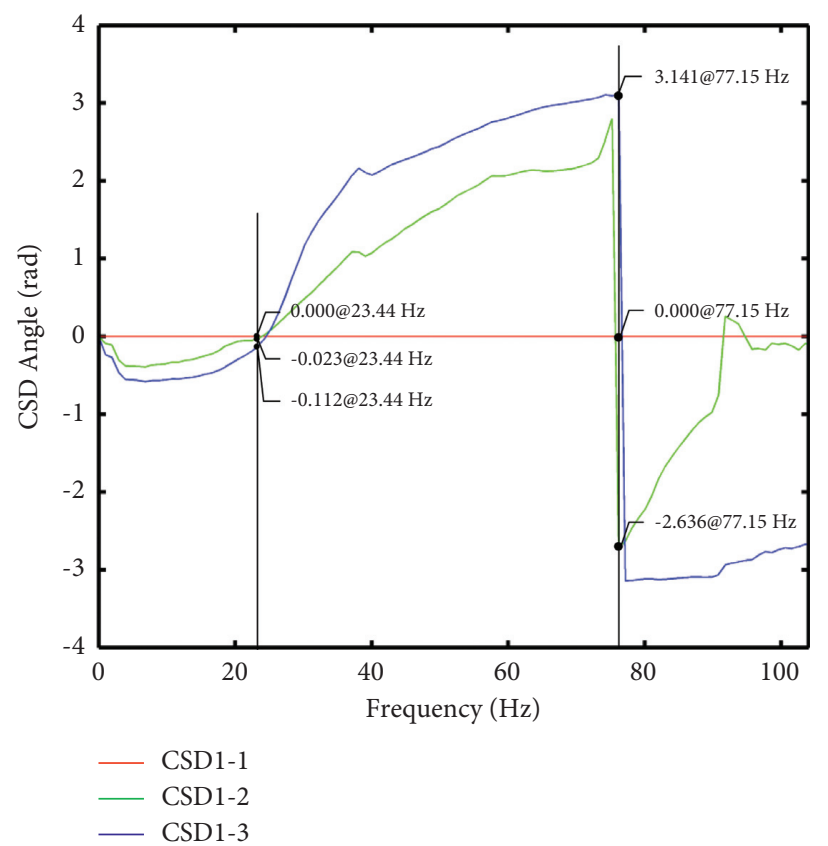

Figure 8: Phase angle curve of the power spectrum.

second component in the first mode. In these three sensitivities, the absolute value of the sensitivity of $\varphi_{2}^{(1)}$ for $m_{2}$ is about $30 \%$ larger than that for $m_{1}$ and $m_{3}$. Surveying the data vertically in Table 4 , the absolute values of the sensitivity $\varphi_{1}^{(1)}, \varphi_{2}^{(1)}$, and $\varphi_{3}^{(1)}$ for $m_{2}$ are much larger than those for $m_{1}$ and $m_{3}$. The absolute values of sensitivity of $\varphi_{1}^{(1)}$ and $\varphi_{3}^{(1)}$ for $m_{1}$ and $m_{3}$ are much smaller than the other sensitivities. This means that changing the local mass of the first and third correction planes (discs) has little effect on the first mode while changing the local mass of the second correction plane 
TABLE 2: Rotor mode shapes normalized by the maximum.

\begin{tabular}{lcccr}
\hline & 1st magnitude & 1st angle & 2nd magnitude & 2nd angle \\
\hline Plane 1 & 0.85 & $0.0^{\circ}$ & 1.00 & $0.0^{\circ}$ \\
Plane 2 & 1.00 & $-1.3^{\circ}$ & 0.09 & $-151.1^{\circ}$ \\
Plane 3 & 0.94 & $-6.4^{\circ}$ & 0.86 & $179.9^{\circ}$ \\
\hline
\end{tabular}

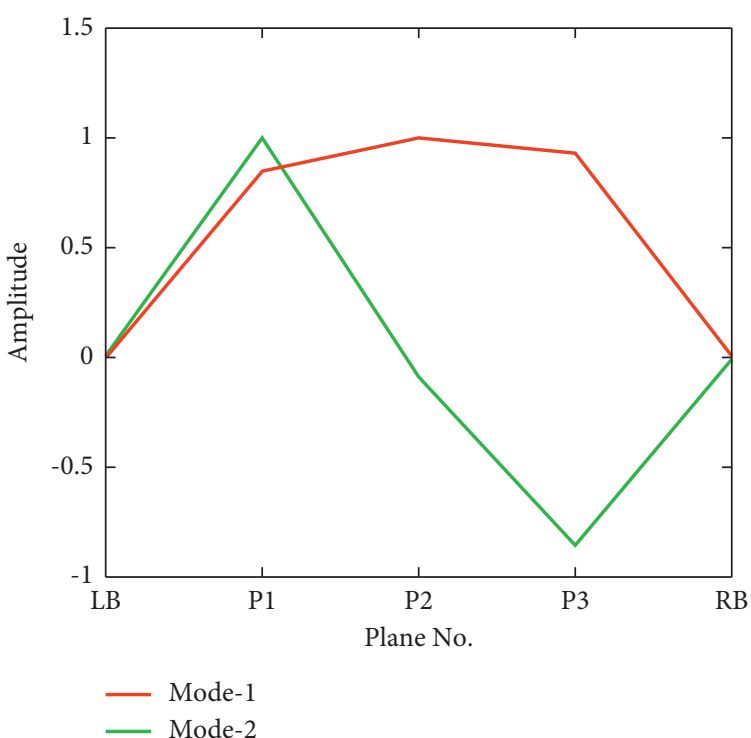

Figure 9: Mode shapes of the rotor.

TABLE 3: Sensitivity matrix for mass changes in the three planes.

\begin{tabular}{lccr}
\hline Sensitivity & $m_{1}$ & $m_{2}$ & $m_{3}$ \\
\hline$\varphi_{1}^{(1)}$ & -0.0521 & -0.1342 & -0.0481 \\
$\varphi_{2}^{(1)}$ & -0.1185 & -0.1562 & -0.1189 \\
$\varphi_{3}^{(1)}$ & -0.0480 & -0.1342 & -0.0436 \\
$\varphi_{1}^{(2)}$ & -0.9497 & -0.0395 & -1.0158 \\
$\varphi_{2}^{(2)}$ & -0.6605 & -0.0430 & -0.6663 \\
$\varphi_{3}^{(2)}$ & -0.9823 & -0.0398 & -1.0536 \\
\hline
\end{tabular}

TABle 4: Comparison of different methods for the rotor balancing at the first critical speed.

\begin{tabular}{|c|c|c|c|c|c|c|}
\hline \multirow[b]{2}{*}{ Correction plane } & \multicolumn{3}{|c|}{ Conventional MBM } & \multicolumn{3}{|c|}{ Optimization approach } \\
\hline & Plane1 & Plane 2 & Plane 3 & Plane 1 & Plane 2 & Plane 3 \\
\hline Initial amplitude $(\mathrm{mm})$ & 0.1408 & 0.1676 & 0.1525 & 0.1408 & 0.1676 & 0.1525 \\
\hline Initial phase $\left(^{\circ}\right)$ & -31.4 & -30.8 & -31.1 & -31.4 & -30.8 & -31.1 \\
\hline Correction mass (g) & 0.45 & 0.37 & 0.44 & 1 & 1.1 & 1 \\
\hline Correction phase $\left(^{\circ}\right)$ & 150.0 & 150.0 & 150.0 & 1 & 150.0 & 1 \\
\hline Actual installation phase $\left(^{\circ}\right)$ & 157.5 & 157.5 & 157.5 & 1 & 157.5 & 1 \\
\hline Residual amplitude (mm) & 0.1011 & 0.1142 & 0.1311 & 0.0821 & 0.0872 & 0.0574 \\
\hline Balancing rate & $28 \%$ & $32 \%$ & $14 \%$ & $42 \%$ & $48 \%$ & $63 \%$ \\
\hline Average balancing rate & $25 \%$ & $51 \%$ & & & & \\
\hline
\end{tabular}

(disc) has a significant effect on the first mode. This result can be clearly shown in Figure 10.

Surveying the data about the second mode horizontally in Table 3, the changes of $m_{1} . m_{2}$ and $m_{3}$ have a relatively large influence on the components $\varphi_{1}^{(2)}$ and $\varphi_{3}^{(2)}$ in the second mode. The sensitivities of $\varphi_{1}^{(2)}$ and $\varphi_{3}^{(2)}$ for $m_{1}$ and $m_{3}$ are almost the same. Taking the data in the first row as an example, the absolute values of the sensitivity $\varphi_{1}^{(2)}$ for $m_{1}$ and $m_{3}$ are about 25 times larger than the sensitivity $\varphi_{1}^{(2)}$ for $m_{2}$. Surveying the data vertically in Table 5, the absolute 


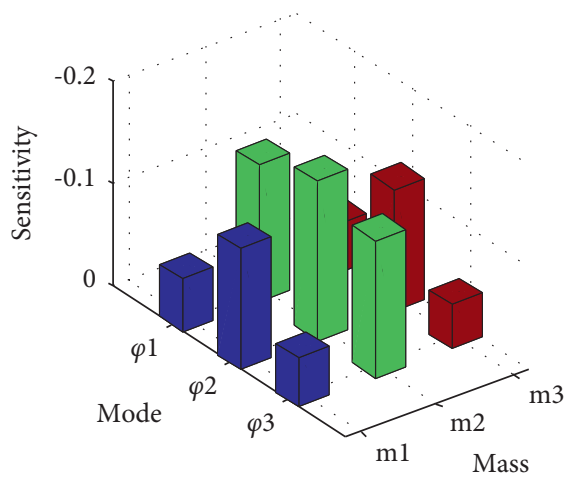

Figure 10: Histogram of the first mode sensitivity matrix.

TABLE 5: Comparison of different methods for the rotor balancing at the second critical speed.

\begin{tabular}{|c|c|c|c|c|c|c|}
\hline \multirow[b]{2}{*}{ Correction plane } & \multicolumn{3}{|c|}{ Conventional MBM } & \multicolumn{3}{|c|}{ Optimization approach } \\
\hline & Plane 1 & Plane 2 & Plane 3 & Plane 1 & Plane 2 & Plane 3 \\
\hline Initial amplitude $(\mathrm{mm})$ & 0.1138 & 0.0431 & 0.1363 & 0.1138 & 0.0431 & 0.1363 \\
\hline Initial phase $\left({ }^{\circ}\right)$ & 152.1 & -42.3 & -41.5 & 152.1 & -42.3 & -41.5 \\
\hline Correction mass (g) & 0.88 & 1.06 & 0.84 & 0.7 & 1 & 0.8 \\
\hline Correction phase $\left(^{\circ}\right)$ & -28.0 & 138.0 & 138.0 & -28.0 & 1 & 138.0 \\
\hline Actual installation phase $\left({ }^{\circ}\right)$ & 337.5 & 135.0 & 135.0 & 337.5 & 1 & 135.0 \\
\hline Residual amplitude (mm) & 0.0834 & 0.0420 & 0.0980 & 0.0341 & 0.0427 & 0.0780 \\
\hline Balancing rate & $27 \%$ & $3 \%$ & $28 \%$ & $70 \%$ & $3 \%$ & $43 \%$ \\
\hline Average balancing rate & $19 \%$ & $39 \%$ & & & & \\
\hline
\end{tabular}

sensitivity values of the three mode components for $m_{2}$ are much smaller than those for $m_{1}$ and $m_{3}$. The sensitivity of $\varphi_{2}^{(2)}$ for $m_{2}$ has the largest absolute value in line 2 , which is 0.0430 . The sensitivity of $\varphi_{2}^{(2)}$ for $m_{1}$ has the smallest absolute value in line 1 and line 3 , which is 0.6605 . The latter is about 16 times larger than the former. This means that changing the local mass of the first and third correction planes has a subequal influence on the second mode shape, which is more considerable than changing the local mass of the second plane. This result can be clearly seen in Figure 11.

5.4. Experimental Results of the Rotor Balancing. After lowspeed balancing, the rotor can pass two critical speeds safely. However, the vibration of the rotor is still high at critical speeds. Therefore, the rotor needs high-speed balancing at critical speeds next. The initial vibration amplitude and the phase angle of the rotor at these critical speeds are extracted from the time domain signals while the rotor is speeding up, as shown in Figure 6. The phase angles of the vibrations for the three discs are calculated based on the pulse signal of the photoelectric sensor.

The optimization analysis shows that balancing the vibration at the first critical speed only requires operating disc 2 ; balancing the vibration at the second critical speed requires operating disc 1 and disc 3 concurrently. Since the first mode shape sensitivities of the mass changes on disc 1 and disc 3 are relatively low, the second mode shape has an extremely low sensitivity for the mass changes on disc 2 . That is, disc 1 and disc 3 have less effect than disc 2 in the first mode. Disc 2 has less effect than disc 1 and disc 3 on the second mode. According to this strategy, the optimized balancing method mentioned above is used to rebalance the rotor.

The data for balancing by using the conventional MBM and the optimization approach are shown in Tables 4 and 5 . Table 4 shows the comparison of the two methods at the first critical speed of the rotor. Table 5 shows the second critical speed.

After balancing the flexible rotor with the conventional MBM, the vibration amplitude of each plane has been reduced. Unfortunately, the reduction of the vibration amplitude in the three discs is not obvious. The highest balancing rate of the first critical speed is only $32 \%$. The average balancing rate in the first critical speed is only $25 \%$. After the correction planes have been optimized, the balancing rate has improved. The maximum balancing rate at the first critical speed is $63 \%$. The average balancing rate is $51 \%$, which is twice as much as that before optimization. From Table 5, it can also be seen that the optimization approach at the second critical speed of the rotor also has a higher balance accuracy than the traditional method.

The vibration response plots of the three discs while the rotors are speeding up are presented in Figure 12. In this figure, the balancing results before balancing, for the conventional MBM and for the optimization approach for the rotor are shown. It is worth mentioning that the vibration response of disc 2 at the second critical speed changes slightly before and after balancing. This is because the second disk is at the node of the second vibration mode of the rotor. It is obvious from the figure that the optimization approach has a better balance effect. 


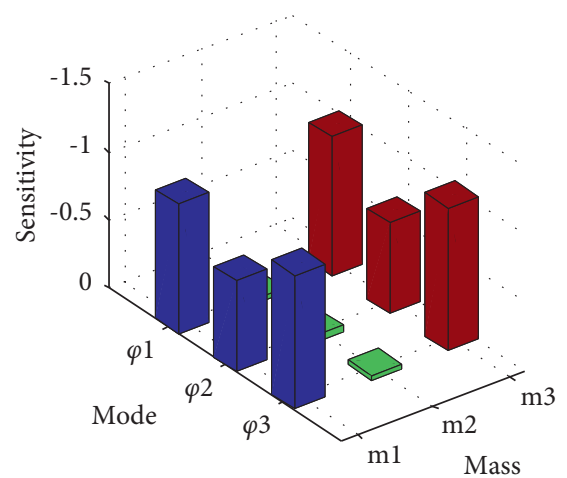

FIGURE 11: Histogram of the second mode sensitivity matrix.

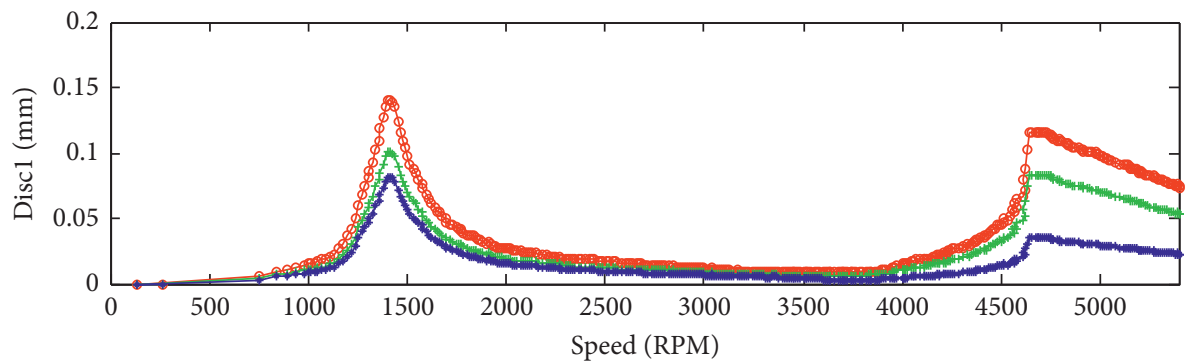

$\rightarrow$ Before Balancing

- Conventional MBM

$\rightarrow$ Optimization Approach

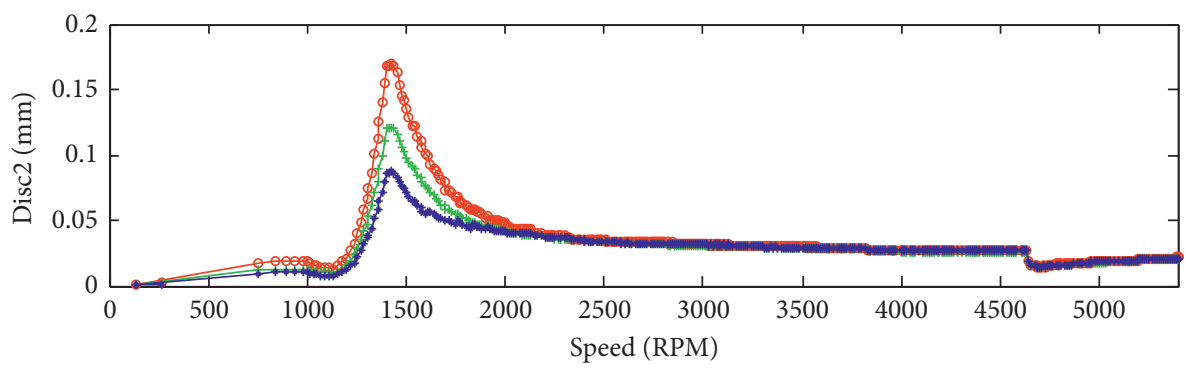

$\rightarrow$ Before Balancing

- Conventional MBM

$\rightarrow$ Optimization Approach

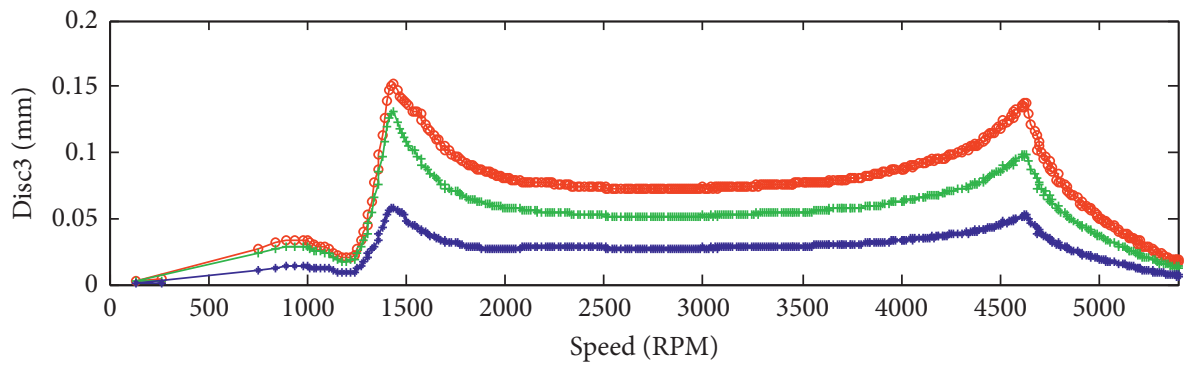

- Before Balancing

$\rightarrow$ Conventional MBM

$\rightarrow$ Optimization Approach

Figure 12: Vibration response of three discs during speeding up. 


\section{Conclusions}

This paper proposes an optimized balancing approach to balance a flexible rotor at critical speeds. The critical speeds and mode shapes of the rotor are obtained with the experimental modal analysis method using the vibration response while the rotor is speeding up. Furthermore, the optimized selection of the rotor correction planes is obtained in the rotor balancing strategy by means of the vibration mode shape sensitivity analysis for the mass changes. The mode shapes of the rotor are used to calculate the orthogonal trial masses in balancing. Then the influence coefficients of the orthogonal trial weights in each mode are calculated with experimentation. The correction masses are obtained to balance the rotor. In every operation of the rotor, it is safe for the operator to use the vibration response signal of the rotor while the rotor is speeding up. Compared to the conventional MBM, the balancing approach proposed in this work is able to achieve better accuracy. This approach is expected to be used in the accurate balancing variety of flexible rotors, especially for rotor balancing in the field.

\section{Data Availability}

The data used to support the findings of this study are included within the article.

\section{Conflicts of Interest}

The authors declare that they have no conflicts of interest.

\section{Acknowledgments}

This study was supported by the National Natural Science Foundation of China (Grant no. 11872045) and the Major Special Basic Research Projects for Aeroengines and Gas Turbines (Grant no. 2017-IV-0008-0045). The authors thank LetPub (http://www.letpub.com) for its linguistic assistance during the preparation of this manuscript.

\section{References}

[1] L. Li, S. Cao, J. Li, R. Nie, and L. Hou, "Review of rotor balancing methods," Machines, vol. 9, no. 5, p. 89, 2021.

[2] L. Hou and S. Cao, "Evaluation method for vibration measurement on casing in aeroengine: theoretical analysis and experimental study," Shock and Vibration, vol. 2019, Article ID 1648709, 11 pages, 2019.

[3] J. Yao, F. Yang, Y. Su, F. Scarpa, and J. Gao, "Balancing optimization of a multiple speeds flexible rotor," Journal of Sound and Vibration, vol. 480, Article ID 115405, 2020.

[4] G. Ranjan and R. Tiwari, "On-site high-speed balancing of flexible rotor-bearing system using virtual trial unbalances at slow run," International Journal of Mechanical Sciences, vol. 183, Article ID 105786, 2020.

[5] S. Tresser, A. Dolev, and I. Bucher, "Dynamic balancing of super-critical rotating structures using slow-speed data via parametric excitation," Journal of Sound and Vibration, vol. 415, pp. 59-77, 2017.
[6] S. Zhong, L. Li, H. Chen, and Z. Lu, "A novel balancing method for rotor using unsupervised deep learning," Shock and Vibration, vol. 2021, Article ID 1800164, 18 pages, 2021.

[7] ISO Standard 11342, Methods and Criteria for the Mechanical Balancing of Flexible Rotors, ISO Standard 11342, Geneva, Switzerland, 1998.

[8] S. Shiyu Zhou and J. Jianjun Shi, "Active balancing and vibration control of rotating machinery: a survey," The Shock and Vibration Digest, vol. 33, no. 5, pp. 361-371, 2001.

[9] A. G. Parkinson, "Balancing of rotating machinery," Proceedings of the Institution of Mechanical Engineers - Part C: Mechanical Engineering Science, vol. 205, no. 1, pp. 53-66, 1991.

[10] W. C. Foiles, P. E. Allaire, and E. J. Gunter, "Review: rotor balancing," Shock and Vibration, vol. 5, no. 5-6, pp. 325-336, 1998.

[11] M. S. Darlow, "Balancing of high-speed machinery: theory, methods and experimental results," Mechanical Systems and Signal Processing, vol. 1, no. 1, pp. 105-134, 1987.

[12] R. Ye, L. Wang, X. Hou, Z. Luo, and Q. Han, "Balancing method without trial weights for rotor systems based on similitude scale model," Frontiers of Mechanical Engineering, vol. 13, no. 4, pp. 571-580, 2018.

[13] J. M. Tessarzik, "Flexible rotor balancing by the influence coefficient method: multiple critical speeds with rigid or flexible supports," NASA Contractor Report NASA CR-2553, 1975.

[14] W. J. Anderson, R. H. Badgley, and J. M. Tessarzik, "Flexible rotor balancing by the exact point- speed influence coefficient method," Journal of Engineering for Industry, vol. 94, no. 1, 1971.

[15] T. P. Goodman, "A least-squares method for computing balance corrections," Journal of Engineering for Industry, vol. 86, no. 3, pp. 273-277, 1964.

[16] Y. Kang, C.-P. Liu, and G.-J. Sheen, "A modified influence coefficient method for balancing unsymmetrical rotor-bearing systems," Journal of Sound and Vibration, vol. 194, no. 2, pp. 199-218, 1996.

[17] B. Xu, L. Qu, and R. Sun, "The optimization technique-based balancing of flexible rotors without test runs," Journal of Sound and Vibration, vol. 238, no. 5, pp. 877-892, 2000.

[18] M. Deepthikumar, A. Sekhar, and M. Srikanthan, "Balancing of flexible rotor with bow using transfer matrix method," Journal of Vibration and Control, vol. 20, no. 2, pp. 225-240, 2014.

[19] L. P. Grobel, "Balancing turbine-generator rotors," Journal of the American Society for Naval Engineers, vol. 65, no. 4, pp. 868-874, 1953.

[20] W. Kellenberger, "Balancing flexible rotors on two generally flexible bearings," Brown Boveri Review, vol. 54, no. 9, pp. 603-617, 1967.

[21] M. Bertoneri and P. Forte, "Turbomachinery high speed modal balancing: modeling and testing of scale rotors," in Proceedings of the 9th IFToMM International Conference on Rotor Dynamics, pp. 15-25, Cassino, Italy, May 2015.

[22] M. Villafane Saldarriaga, V. Steffen, J. Der Hagopian, and J. Mahfoud, "On the balancing of flexible rotating machines by using an inverse problem approach," Journal of Vibration and Control, vol. 17, no. 7, pp. 1021-1033, 2010.

[23] Y. A. Khulief and M. A. Mohiuddin, "On the dynamic analysis of rotors using modal reduction," Finite Elements in Analysis and Design, vol. 26, no. 1, pp. 41-55, 1997.

[24] X. Li, L. Zheng, and Z. Liu, "Balancing of flexible rotors without trial weights based on finite element modal analysis," 
Journal of Vibration and Control, vol. 19, no. 3, pp. 461-470, 2012.

[25] Y. Zhang, M. Li, H. Yao, Y. Gou, and X. Wang, "A modalbased balancing method for a high-speed rotor without trial weights," Mechanical Sciences, vol. 12, no. 1, pp. 85-96, 2021.

[26] J. K. Sinha, A. W. Lees, and M. I. Friswell, "Estimating unbalance and misalignment of a flexible rotating machine from a single run-down," Journal of Sound and Vibration, vol. 272, no. 3, pp. 967-989, 2004.

[27] I. Bucher and D. J. Ewins, "Modal analysis and testing of rotating structures," Philosophical Transactions of the Royal Society of London,Series A: Mathematical, Physical and Engineering Sciences, vol. 359, no. 1778, pp. 61-96, 2001.

[28] S. Cao, W. Zhang, and L. Xiao, Structural Vibration Modal Analysis: Theory, experiment and Application, Tianjin University Press, Tianjin, China, 2014. 